\title{
On the Risk of Ruin in a SIS Type Epidemic
}

\author{
Claude Lefèvre $^{1}$ (D) Matthieu Simon ${ }^{2,3}$
}

Received: 12 May 2021 / Revised: 11 October 2021 / Accepted: 13 December 2021 /

Published online: 8 January 2022

() The Author(s), under exclusive licence to Springer Science+Business Media, LLC, part of Springer Nature 2022

\begin{abstract}
The paper deals with the problem of possible ruin when providing insurance coverage for an epidemic. The model studied is an SIS type epidemic which generalizes the well-known logistic model. Contractually, the premiums are paid by susceptible people while the care costs are reimbursed to infected people via an annuity or a lump-sum benefit. Our goal is to determine the distribution of the main statistics of the ruin when it occurs during the epidemic. The case where the reserve alternates between normal and epidemic episodes is also discussed using a Brownian modeling of the reserve. Finally, some of the results are illustrated for two particular SIS epidemic models.
\end{abstract}

Keyword Birth-and-death epidemic model · Insurance coverage $\cdot$ Ruin statistics · Brownian regenerative process

AMS Subject Classifications $91 \mathrm{G} 05 \cdot 92 \mathrm{D} 30 \cdot 60 \mathrm{~J} 28$

\section{Introduction}

There is a vast mathematical literature on epidemic models and their applications. Much material on the stochastic approach can be found in the books by Daley and Gani (1999), Andersson and Britton (2000) and Britton and Pardoux (eds.) (2019). Two central classes of models concern epidemics of the SIR (Susceptible-Infected-Removed) type and the SIS (Susceptible-Infected-Susceptible) type. In short, in the first case an infected is permanently eliminated by death or vaccination, while in the second case an infected recovers and becomes again exposed to the disease.

Claude Lefèvre

Claude.Lefevre@ulb.be

Matthieu Simon

Matthieu.Simon@umons.ac.be

1 Département de Mathématique, Université Libre de Bruxelles, Campus Plaine C.P. 210,

Bruxelles B-1050, Belgium

2 Departament de Matemàtica Econòmica, Universitat de Barcelona, Financera i Actuarial, 690 Avinguda Diagonal, Barcelona E-08034, Spain

3 Département de Mathématique, Université de Mons, 20 Place du Parc, Mons B-7000, Belgium 
This paper focuses on a stochastic SIS epidemic process that spreads in a closed population of total size $N$. Let $\left(S_{t}, I_{t}\right)$ be the number of susceptibles and infectives present at time $t$. By definition, $S_{t}+I_{t}=N$ for all $t$. The evolution of $S_{t}$ in time is described by a Markovian birth-and-death process whose possible transitions are

$$
\begin{aligned}
& s \rightarrow s-1, \quad \text { at rate } \beta(s), \\
& s \rightarrow s+1, \quad \text { at rate } \mu(s) .
\end{aligned}
$$

So, $\beta(s)$ represents the global infection rate on $s$ susceptibles and $\mu(s)$ the global recovery rate for $i=N-s$ infectives. Of course, we assume $\beta(0)=0($ since $s=0)$ and $\mu(N)=0$ (since $s=N$ ). Moreover, it is natural to take $\beta(N)=0$ (since $i=0$ ), which makes $N$ an absorbing state for the susceptibles. All the other rates are chosen strictly positive.

A classic special case is the logistic SIS epidemic in which $\beta(s)=\beta s(N-s)$ and $\mu(s)=v(N-s)$ for some constants $\beta, v>0$. Here, the infection rate is proportional to the number of possible infectious contacts and the recovery rate to the number of infected. This model has received considerable attention, especially due to the existence of a quasistationary state. We refer the reader to the book by Nåsell (2011) and to numerous articles including Andersson and Djehiche (1998), van Doorn and Pollett (2013) and Clancy and Tjia (2018). Various extensions of this model were also discussed, e.g. by Nåsell (1999) and Clancy (2005).

The problem that we will study concerns the insurance coverage of a birth-and-death epidemic process (1). We will thus combine ruin theory and epidemiology, two fields which are not generally associated with each other. Indeed, very little work has to date been devoted to actuarial applications of epidemic models. In addition, they were all carried out for infectious diseases of the SIR type. Thus, the seminal article by Feng and Garrido (2011) dealt with the deterministic version of a well-known model called the general epidemic. A stochastic approach for a Markovian version of this model was then developed by Lefèvre et al. (2017). The related question of the possible occurrence of ruin has just been investigated by Lefèvre and Simon (2021). Very recently, Feng et al. (2022) reviewed basic compartmental epidemic models with several actuarial applications.

Measuring the risk of ruin and its consequences is a central subject of traditional mathematical theory of insurance. A fairly comprehensive survey of the literature is provided by the book of Asmussen and Albrecher (2010). The reader is also referred e.g. to the books by Dickson (2017) and Schmidli (2018). Our aim will be to examine the problem of insurance coverage and possible ruin for an epidemic model that has precisely the SIS schema described above.

The paper is organized as follows. In Section 2, we derive the distribution of several variables that influence the cost of insurance coverage for a SIS type epidemic (Propositions 2.1, 2.2 and their Corollaries). In the next two sections, we consider two different insurance plans to cover medical costs caused by the epidemic. Premiums are collected from policyholders as long as they are in good heath (susceptible), and financial compensation is provided to policyholders whenever they catch the disease (infected). In Section 3, the company reimburses the care of infectious people for the duration of the treatment (annuity benefit). In Section 4, a compensation of random (exponential) amount is paid to each infected as soon as they recover from the disease (lump-sum benefit). We mainly focus on the ruin of the company when it occurs before the infection is over. Thus, we determine the joint distribution of the time of ruin $T_{r}$, the total number of contaminations $N_{T_{r}}$ and the final population state $S_{T_{r}}$ (Propositions 3.1, 3.2 and 4.1, 4.2). In Section 5, we consider a risk process over a large time scale so that it can now alternate between two regimes, normal or 
epidemic. This leads us to introduce a regenerative extension of the model using a Brownian reserve process. Here too, we obtain the distribution of the ruin statistics above together with the number $\mathcal{J}_{T_{r}}$ of epidemics triggered before ruin (Proposition 5.1). In Section 6, we illustrate and comment on some of the results for two particular epidemic models of SIS type.

\section{Insurance Cost Components}

By virtue of (1), the susceptible process $\left\{S_{t}, t \geq 0\right\}$ is Markovian with state space $\{0, \ldots, N\}$ and generator

$$
\left[\begin{array}{ccccccc}
-\gamma(0) & \mu(0) & 0 & 0 & \cdots & 0 & 0 \\
\beta(1) & -\gamma(1) & \mu(1) & 0 & \cdots & 0 & 0 \\
0 & \beta(2) & -\gamma(2) & \mu(2) & \cdots & 0 & 0 \\
\vdots & \vdots & \vdots & \vdots & \ddots & \vdots & \vdots \\
0 & 0 & 0 & 0 & \cdots & -\gamma(N-1) & \mu(N-1) \\
0 & 0 & 0 & 0 & \cdots & 0 & 0
\end{array}\right],
$$

where $\gamma(s) \equiv \mu(s)+\beta(s)$ for $0 \leq s \leq N$. Obviously, the last state $N$ is absorbing, and since it is a finite Markov chain, $N$ will be attained after a finite time

$$
T=\inf \left\{t \geq 0: S_{t}=N\right\} .
$$

We introduce two specific random variables that will play a key role in the insurance context. On the one hand, the variable $\mathcal{N}_{T}$ which counts the new contaminations made up to $T$ (i.e. the total number of transitions $s \rightarrow s-1$ until absorption). This statistic gives a measure of the virulence of the contamination until the end of infection. On the other hand, the variable $\mathcal{H}_{T}$ which is defined as

$$
\mathcal{H}_{T}=\int_{0}^{T} h\left(S_{t}\right) d t
$$

where $h$ is any positive real function defined on $\{0, \ldots, N-1\}$. This statistic allows us to assess the total costs or revenues incurred by the susceptibles. Note that $T$ is a special case of $\mathcal{H}_{T}$ with the choice $h(s)=1$ for all $s$.

Let us derive the joint Laplace transform of these two variables when the initial state is $S_{0}=s(<N)$.

Proposition 2.1 For $\theta \geq 0$ and $z \in(0,1]$,

$$
\mathbb{E}\left[e^{-\theta \mathcal{H}_{T}} z^{\mathcal{N}_{T}} \mid S_{0}=s\right]=\prod_{l=s}^{N-1} g_{l}(\theta, z), \quad 0 \leq s \leq N-1,
$$

where the $g_{l}(\theta, z)$ satisfy the recursion

$$
g_{l}(\theta, z)=\frac{\mu(l)}{\gamma(l)+\theta h(l)}+\frac{\beta(l)}{\gamma(l)+\theta h(l)} z g_{l-1}(\theta, z) g_{l}(\theta, z), \quad 0 \leq l \leq N-1,
$$

with $g_{-1}(\theta, z) \equiv 0$. 
Proof Let $\tau_{l}$ be the first-passage time of the susceptible process at the height $l$, i.e.

$$
\tau_{l}=\inf \left\{t \geq 0: S_{t}=l\right\}, \quad 0 \leq l \leq N,
$$

(so, $\tau_{N}=T$ ), and consider the expectations

$$
g_{l}(\theta, z)=\mathbb{E}\left[e^{-\theta \mathcal{H}_{\tau_{l+1}}} z^{\mathcal{N}_{\tau_{l+1}}} \mid S_{0}=l\right], \quad 0 \leq l \leq N-1
$$

Now, starting in state $s$, the susceptible process must go through the states $s+1, \ldots$, $N-1$ before being absorbed into $N$. From (4) and (8), we then directly obtain the equality (5). To get the recursion (6), we condition in (8) with respect to the duration $v$ before the first change of state. Using (2) and (4), we find that

$$
g_{l}(\theta, z)=\int_{0}^{\infty} e^{-[\gamma(l)+\theta h(l)] v} \mu(l) d v+\int_{0}^{\infty} e^{-[\gamma(l)+\theta h(l)] v} \beta(l) d v z g_{l-1}(\theta, z) g_{l}(\theta, z) .
$$

The reasoning for the second integral is that the first change of state is the infection of a susceptible among the $l$ present, and the number of susceptible must then increase twice before reaching $l+1$. The formula (6) follows after integration.

In particular, the mean of $\mathcal{N}_{T}$ and $\mathcal{H}_{T}$ easily follow from (5), (6).

Corollary 2.1 For $0 \leq s \leq N-1$,

$$
\begin{gathered}
\mathbb{E}\left[\mathcal{N}_{T} \mid S_{0}=s\right]=\sum_{l=s}^{N-1} \sum_{k=1}^{l}\left(\prod_{j=k}^{l} \frac{\beta(j)}{\mu(j)}\right), \\
\mathbb{E}\left[\mathcal{H}_{T} \mid S_{0}=s\right]=\sum_{l=s}^{N-1} \sum_{k=0}^{l} \frac{h(k)}{\mu(k)}\left(\prod_{j=k+1}^{l} \frac{\beta(j)}{\mu(j)}\right) .
\end{gathered}
$$

Proof For brevity, we will only derive (10). From (5), we have

$$
\mathbb{E}\left[\mathcal{H}_{T} \mid S_{0}=s\right]=-\left[\frac{d}{d \theta} \prod_{l=s}^{N-1} g_{l}(\theta, z)\right]_{\theta=0, z=1}=-\sum_{l=s}^{N-1}\left[\frac{d}{d \theta} g_{l}(\theta, z)\right]_{\theta=0, z=1},
$$

since $g_{l}(0,1)=1$ by (8). Set $x_{l}=-\left[d g_{l}(\theta, z) / d \theta\right]_{\theta=0, z=1}$. Differentiating (6), we see that the $x_{l}$ satisfy the recursion

$$
\mu(l) x_{l}=h(l)+\beta(l) x_{l-1}, \quad 0 \leq l \leq N-1,
$$

with $x_{-1} \equiv 0$. The solution of this recursion is found to be

$$
x_{l}=\sum_{k=0}^{l} \frac{h(k)}{\mu(k)}\left(\prod_{j=k+1}^{l} \frac{\beta(j)}{\mu(j)}\right)
$$

under the convention $\prod_{j=l+1}^{l}=1$. Inserting (12) into (11) then gives (10).

Another quantity used for insurance cost is the probability that starting from $S_{0}=n$, the number of susceptibles reaches any given level $s<n$ before the end of the epidemic (see Section 4). In order to determine it, we will first obtain the Laplace transform of the vector 
$\left(\mathcal{N}_{\tau_{s}}, \mathcal{H}_{\tau_{s}}\right)$ with the constraint $\tau_{s}<T$ where $\tau_{s}$ is defined by (7) and $s<n$. The reasoning is similar to that followed for Proposition 2.1. As usual, $\mathbb{1}_{A}$ denotes the indicator variable for a random event $A$.

Proposition 2.2 For $0 \leq s \leq n-1$,

$$
\mathbb{E}\left[e^{-\theta \mathcal{H}_{\tau_{s}}} z^{\mathcal{N}_{\tau_{s}}} \mathbb{1}_{\tau_{s}<T} \mid S_{0}=n\right]=\prod_{l=s+1}^{n} q_{l}(\theta, z),
$$

where the $q_{l}(\theta, z)$ satisfy the recursion

$$
q_{l}(\theta, z)=z \frac{\beta(l)}{\gamma(l)+\theta h(l)}+\frac{\mu(l)}{\gamma(l)+\theta h(l)} q_{l}(\theta, z) q_{l+1}(\theta, z), \quad 1 \leq l \leq N-1,
$$

with $q_{N}(\theta, z) \equiv 0$.

Proof Consider the expectations

$$
q_{l}(\theta, z)=\mathbb{E}\left[e^{-\theta \mathcal{H}_{\tau_{l-1}}} z^{\mathcal{N}_{\tau_{l-1}}} \mathbb{1}_{\tau_{l-1}<T} \mid S_{0}=l\right], \quad 1 \leq l \leq N-1 .
$$

To reach $s$ from $n$, the susceptible process must go through the states $n-1, \ldots, s+1$ before absorption. So, (13) directly follows from (15). For the recursion (14), we condition in (15) with respect to the length $v$ before the first state change. This gives

$$
q_{l}(\theta, z)=\int_{0}^{\infty} e^{-[\gamma(l)+\theta h(l)] v} \beta(l) z d v+\int_{0}^{\infty} e^{-[\gamma(l)+\theta h(l)] v} \mu(l) d v q_{l+1}(\theta, z) q_{l}(\theta, z),
$$

which yields the desired result.

In particular, we deduce an explicit expression for the first-passage probabilities from $n$ to $s<n$.

Corollary 2.2 For $0 \leq s \leq n-1$,

$$
\mathbb{P}\left(\tau_{s}<T \mid S_{0}=n\right)=\prod_{l=s+1}^{n} q_{l},
$$

where the $q_{l}$ are given by

$$
q_{l}=\sum_{k=1}^{N-l}\left(\prod_{j=l}^{N-k} \frac{\beta(j)}{\mu(j)}\right) /\left[1+\sum_{k=1}^{N-l}\left(\prod_{j=l}^{N-k} \frac{\beta(j)}{\mu(j)}\right)\right] .
$$

Proof Define the probabilities

$$
q_{l}=\mathbb{P}\left(\tau_{l-1}<T \mid S_{0}=l\right), \quad 1 \leq l \leq N-1 .
$$

When $\theta=0$ and $z=1, q_{l}(\theta, z)$ reduces to $q_{l}$, so that (13) becomes (16). For (17), consider the recurrence (14) with again $\theta=0$ and $z=1$. This yields

$$
1=\frac{\beta(l)}{\mu(l)}\left(1-q_{l}\right)+1-q_{l}+q_{l} q_{l+1},
$$


with $q_{N} \equiv 0$. In the two sides of this identity, let us substract $q_{l+1}$ and then divide by $\left(1-q_{l}\right)\left(1-q_{l+1}\right)$. Making the change of variable $x_{l}=1 /\left(1-q_{l}\right)$, we get the relation

$$
x_{l}=\frac{\beta(l)}{\mu(l)} x_{l+1}+1, \quad 1 \leq l \leq N-1,
$$

with $x_{N} \equiv 1$. The solution of this recursion is

$$
x_{l}=1+\sum_{k=1}^{N-l}\left(\prod_{j=l}^{N-k} \frac{\beta(j)}{\mu(j)}\right) \text {. }
$$

Substituting (18) into $x_{l}$ gives (17) for $q_{l}$.

\section{Contract with Annuity Benefit}

Consider an epidemic insurance plan in which premiums are collected from policyholders as long as they are in good heath (susceptible), and financial compensation is provided to policyholders, according to certain rules, whenever they catch the disease (infected). In this section, we assume that the company undertakes to reimburse the medical costs of infectious persons for the whole period of treatment.

The Insurance Plan Specifically, the net income rate of the insurance at time $t$ will depend on the population state at time $t$. When $S_{t}=s$, the rate is $c(s)=p(s)-r(s)$ where $p(s)$ represents the rate of premium collection and $r(s)$ the rate of medical reimbursement. A common case is when $c(s)=p s-\rho(N-s)$, which means that the premium is charged at the fixed rate $p$ per susceptible and the reimbursement is given at the fixed rate $\rho$ per infected. Of course, the function $c(s)$ can also be non-linear to take account of group effects, for example, in the collection of premiums or the costs of care.

It is natural to suppose that $c(s)$ increases with $s$, with first negative and then positive values. So, $c\left(s_{1}\right) \geq c\left(s_{2}\right)$ if $s_{1}>s_{2}$, and there exists $s_{*} \in\{1, \ldots, N-1\}$ such that $c(s)<0$ for $s<s_{*}$ and $c(s)>0$ for $s \geq s_{*}$. For simplicity, we take $c(s) \neq 0$ for all $s$, but this can be easily lifted.

The coverage rate $r(s)$ is normally determined or estimated by the company. On the basis of this amount, the company calculates a premium rate $p(s)$ for a given premium structure, typically $p(s)=p s$. For this, a criterion often used is the Equivalence Principle in life insurance, which requires that $\mathbb{E}[$ benefit outgo $]=\mathbb{E}[$ premium income $]$. Thus, according to this principle, the fair value of the premium is set from the equality

$$
\mathbb{E}\left[\int_{0}^{T} p\left(S_{t}\right) d t\right]=\mathbb{E}\left[\int_{0}^{T} r\left(S_{t}\right) d t\right] .
$$

From (10), we can rewrite (19) for $S_{0}=n(<N)$ as

$$
\sum_{l=n}^{N-1} \sum_{k=0}^{l} \frac{p(k)}{\mu(k)}\left(\prod_{j=k+1}^{l} \frac{\beta(j)}{\mu(j)}\right)=\sum_{l=n}^{N-1} \sum_{k=0}^{l} \frac{r(k)}{\mu(k)}\left(\prod_{j=k+1}^{l} \frac{\beta(j)}{\mu(j)}\right),
$$

and then deduce a function $p(s)$ with the desired structure.

In particular, if $p(s)=p s$, the fair premium level $p_{*}$ given by (20) is 


$$
p_{*}=\left[\sum_{l=n}^{N-1} \sum_{k=0}^{l} \frac{r(k)}{\mu(k)}\left(\prod_{j=k+1}^{l} \frac{\beta(j)}{\mu(j)}\right)\right] /\left[\sum_{l=n}^{N-1} \sum_{k=1}^{l} \frac{k}{\mu(k)}\left(\prod_{j=k+1}^{l} \frac{\beta(j)}{\mu(j)}\right)\right] \text {. }
$$

The Reserve Process To the susceptible process we associate a reserve process $\left\{R_{t}, t \geq 0\right\}$ where $R_{t}$ represents the net capital of the insurance at time $t$. Initially, $R(0)=u \geq 0$. The reserve then evolves continuously over time by adding the premiums received and subtracting the refunds made. Explicitly,

$$
R_{t}=u+\int_{0}^{t} p\left(S_{v}\right) d v-\int_{0}^{t} r\left(S_{v}\right) d v, \quad t \geq 0
$$

So, $R_{t}$ can be positive or not, and increase or not. Its trajectories are continuous and piecewise linear (they are linear if $p$ and $r$ are constant). Until the end time $T, R_{t}$ varies according to $S_{t}$ through the rate of net income $c\left(S_{t}\right)$, i.e.

$$
d R_{t}= \begin{cases}c(s) & \text { if } t<T \text { and } S_{t}=s \\ 0 & \text { if } t \geq T\end{cases}
$$

From now on, we focus our study on the ruin time of the company, defined as $T_{r}=\inf \left\{t>0 \mid R_{t} \leq 0\right\}$, when ruin occurs before the end of the infection $\left(T_{r}<T\right)$. Let $\mathcal{N}_{T_{r}}$ be the total number of contaminations up to time $T_{r}$ and $S_{T_{r}}$ the number of susceptibles at $T_{r}$. Our goal is to determine the expectation

$$
\mathbb{E}\left[e^{-\theta T_{r}} z^{\mathcal{N}_{T_{r}}} \mathbb{1}_{S_{T_{r}}=j, T_{r}<T}\right],
$$

for $\theta \geq 0, z \in(0,1]$ and $0 \leq j \leq s_{*}-1$ since the net rate is necessarily negative at $T_{r}$. This is the Laplace transfiorm of the vector $\left(T_{r}, \mathcal{N}_{T}\right)$ together with the specification of the population state $S_{T_{r}}$ when the ruin actually occurs during the epidemic.

To obtain this transform, we will use the row vectors $\Psi_{s}(\theta, z), s=s_{*}, \ldots, N-1$, of dimension $s_{*}$ whose $j$-th element, $0 \leq j \leq s_{*}-1$, is defined by

$$
\left(\Psi_{s}(\theta, z)\right)_{j}=\mathbb{E}\left[e^{-\theta T_{r}} z^{\mathcal{N}_{T_{r}}} \mathbb{1}_{S_{T_{r}}=j, T_{r}<T} \mid S_{0}=s, R_{0}=0\right] .
$$

Note that the initial reserve here is taken to be 0 . In addition, we introduce the $s_{*} \times s_{*}$ matrix

$$
Q(\theta, z)=\left[\begin{array}{cccccc}
-\frac{\gamma(0)+\theta}{c(0)} & \frac{\mu(0)}{c(0)} & 0 & \cdots & 0 & 0 \\
z \frac{\beta(1)}{c(1)} & -\frac{\gamma(1)+\theta}{c(1)} & \frac{\mu(1)}{c(1)} & \cdots & 0 & 0 \\
0 & z \frac{\beta(2)}{c(2)} & -\frac{\gamma(2)+\theta}{c(2)} & \cdots & 0 & 0 \\
\vdots & \vdots & \vdots & \ddots & \vdots & \vdots \\
0 & 0 & 0 & \cdots & z \frac{\beta\left(s_{*}-1\right)}{c\left(s_{*}-1\right)} & -\frac{\gamma\left(s_{*}-1\right)+\theta}{c\left(s_{*}-1\right)}
\end{array}\right],
$$

and we define the $1 \times s_{*}$ vector $\mathbf{e}=(0, \ldots, 0,1)$.

Proposition 3.1 Let $\theta \geq 0, z \in(0,1]$ and $0 \leq j \leq s_{*}-1$. Starting with $n<s_{*}$ susceptibles and a reserve $u \geq 0$, 


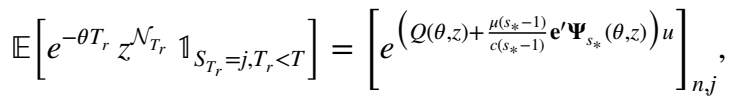

while starting with $n \geq s_{*}$ susceptibles and a reserve $u \geq 0$,

$$
\mathbb{E}\left[e^{-\theta T_{r}} z^{\mathcal{N}_{T_{r}}} \mathbb{1}_{S_{T_{r}}=j, T_{r}<T}\right]=\left[\boldsymbol{\Psi}_{n}(\theta, z) e^{\left(Q(\theta, z)+\frac{\mu\left(s_{*}-1\right)}{c\left(s_{*}-1\right)} \mathbf{e}^{\prime} \boldsymbol{\Psi}_{s_{*}}(\theta, z)\right) u}\right]_{j}
$$

Proof For $x \geq 0$, let $\Phi(\theta, z, x)$ be the $s_{*} \times s_{*}$ matrix of elements

$$
(\Phi(\theta, z, x))_{s, j}=\mathbb{E}\left[e^{-\theta T_{r}} z^{\mathcal{N}_{T_{r}}} \mathbb{1}_{S_{T_{r}}=j, T_{r}<T} \mid S_{0}=s, R_{0}=x\right],
$$

where $0 \leq s, j<s_{*}$. So, it gives the transform of interest when initially, the reserve is $x$ and the number of susceptibles is $s<s_{*}$. Therefore, starting with $S_{0}=n$ susceptibles and a reserve $u \geq 0$, we have

$$
\mathbb{E}\left[e^{-\theta T_{r}} z^{\mathcal{N}_{T_{r}}} \mathbb{1}_{S_{T_{r}}=j, T_{r}<T}\right]= \begin{cases}(\Phi(\theta, z, u))_{n, j} & \text { if } n<s_{*}, \\ \left(\boldsymbol{\Psi}_{n}(\theta, z) \Phi(\theta, z, u)\right)_{j} & \text { if } n \geq s_{*},\end{cases}
$$

using the definition (24).

Now, $\Phi(\theta, z, 0)=I$ and $\Phi(\theta, z, x)$ satisfies $\Phi(\theta, z, x+y)=\Phi(\theta, z, x) \Phi(\theta, z, y)$. Indeed, to reach 0 starting from $x+y$, the reserve process must first reach $y$ and the corresponding probabilities are in $\Phi(\theta, z, x)$; then, the past is forgotten by the strong Markov property and the first passage probabilities at 0 are given by $\Phi(\theta, z, y)$. Therefore, $\Phi(\theta, z, x)$ is of the form

$$
\Phi(\theta, z, x)=e^{U x},
$$

where the matrix $U \equiv U(\theta, z)$ is to be found. For that, we consider (28) and condition on the time of the first change of state. Setting $\kappa(s)=(\gamma(s)+\theta) / c(s)$, we obtain that for $0<s<s_{*}-1$,

$$
\begin{aligned}
\left(e^{U x}\right)_{s, j}= & e^{-\kappa(s) x} \delta_{s, j}+\int_{0}^{x} e^{-\kappa(s) y} \frac{\mu(s)}{c(s)}\left(e^{U(x-y)}\right)_{s+1, j} d y \\
& \quad+\int_{0}^{x} e^{-\kappa(s) y} \frac{\beta(s)}{c(s)} z\left(e^{U(x-y)}\right)_{s-1, j} d y \\
= & e^{-\kappa(s) x}\left(\delta_{s, j}+\int_{0}^{x} e^{\kappa(s) y}\left[\frac{\mu(s)}{c(s)}\left(e^{U y}\right)_{s+1, j}+\frac{\beta(s)}{c(s)} z\left(e^{U y}\right)_{s-1, j}\right] d y\right) .
\end{aligned}
$$

For $s=0$, we get similarly

$$
\left(e^{U x}\right)_{0, j}=e^{-\kappa(0) x}\left(\delta_{0, j}+\int_{0}^{x} e^{\kappa(0) y} \frac{\mu(0)}{c(0)}\left(e^{U y}\right)_{1, j} d y\right),
$$

and for $s=s_{*}-1$,

$$
\begin{gathered}
\left(e^{U x}\right)_{s_{*}-1, j}=e^{\kappa\left(s_{*}-1\right) x}\left(\delta_{s_{*}-1, j}+\int_{0}^{x} e^{\kappa\left(s_{*}-1\right) y} \frac{\beta\left(s_{*}-1\right)}{c\left(s_{*}-1\right)} z\left(e^{U y}\right)_{s_{*}-2, j} d y\right. \\
\left.+\int_{0}^{x} e^{\kappa\left(s_{*}-1\right) y} \frac{\mu\left(s_{*}-1\right)}{c\left(s_{*}-1\right)}\left(\Psi_{S_{*}}(\theta, z) e^{U y}\right)_{j} d y\right)
\end{gathered}
$$


Differentiating (31), (32), (33) with respect to $x$ then taking $x=0$ leads respectively to the relations

$$
\begin{aligned}
& U_{s, j}=-\kappa(s) \delta_{s, j}+\frac{\mu(s)}{c(s)} \delta_{s+1, j}+\frac{\beta(s)}{c(s)} z \delta_{s-1, j}, \quad 0<s<s_{*}-1, \\
& U_{0, j}=-\kappa(0) \delta_{0, j}+\frac{\mu(0)}{c(0)} \delta_{1, j}, \\
& U_{s_{*}-1, j}=-\kappa\left(s_{*}-1\right) \delta_{s_{*}-1, j}+\frac{\mu\left(s_{*}-1\right)}{c\left(s_{*}-1\right)}\left(\Psi_{s}(\theta, z)\right)_{j}+\frac{\beta\left(s_{*}-1\right)}{c\left(s_{*}-1\right)} z \delta_{s_{*}-2, j} .
\end{aligned}
$$

Using (25), we see that a compact form for $U$ is

$$
U=Q(\theta, z)+\frac{\mu\left(s_{*}-1\right)}{c\left(s_{*}-1\right)} \mathbf{e}^{\prime} \boldsymbol{\Psi}_{s_{*}}(\theta, z) .
$$

It remains to insert (30) with (34) in (29) to deduce (26) and (27).

Of course, we now need to determine the vectors $\boldsymbol{\Psi}_{s}(\theta, z)$ defined by (24). They are provided by (35), (36) below.

Proposition 3.2 For $s=s_{*}, \ldots, N-1$,

$$
\Psi_{s}(\theta, z)=\mathbf{e} \prod_{l=s_{*}}^{s} M_{l}(\theta, z),
$$

where the $M_{l}(\theta, z)$ are $s_{*} \times s_{*}$ matrices given recursively by

$$
\begin{array}{r}
M_{l}(\theta, z)=\frac{\beta(l)}{c(l)} z\left(\frac{\gamma(l)+\theta}{c(l)} I-Q(\theta, z)-\frac{\mu\left(s_{*}-1\right)}{c\left(s_{*}-1\right)} \mathbf{e}^{\prime} \boldsymbol{\Psi}_{s_{*}}(\theta, z)-\frac{\mu(l)}{c(l)} M_{l+1}(\theta, z)\right)^{-1}, \\
s_{*} \leq l \leq N-1,
\end{array}
$$

with $M_{N}(\theta, z) \equiv 0$.

Proof For $s_{*} \leq l \leq N-1$, let $\tau_{l}=\inf \left\{t>0 \mid S_{t}=l\right\}$ (as in (7)), and define

$$
m_{l}(\theta, z, x)=\frac{d}{d x} \mathbb{E}\left[e^{-\theta \tau_{l-1}} z^{\mathcal{N}_{\tau_{l-1}}} \mathbb{1}_{R_{\tau_{l-1} \leq x}} \mid S_{0}=l, R_{0}=0\right] .
$$

Now, consider $\left(\boldsymbol{\Psi}_{s}(\theta, z)\right)_{j}, j<s_{*} \leq s \leq N-1$. Conditioning on the level of increase $x$ of the reserve before the first-passage of the population at height $s_{*}-1$, we easily see that

$$
\left(\Psi_{s}(\theta, z)\right)_{j}=\int_{0}^{\infty} m_{s} * m_{s-1} * \cdots * m_{s_{*}}(\theta, z, x)(\Phi(\theta, z, x))_{s_{*}-1, j} d x,
$$

where $*$ denotes the convolution product with respect to $x$ and $\Phi(\theta, z, x)$ is the matrix (28). By $(30), \Phi(\theta, z, x)=\exp (U x)$ so that from (38),

$$
\boldsymbol{\Psi}_{s}(\theta, z)=\mathbf{e} M_{s}(\theta, z) M_{s-1}(\theta, z) \ldots M_{s_{*}}(\theta, z),
$$

where the matrices $M_{l}(\theta, z)$ are given by 


$$
M_{l}(\theta, z)=\int_{0}^{\infty} m_{l}(\theta, z, x) e^{U x} d x
$$

To determine these $M_{l}(\theta, z)$, we consider (37) and condition on the time $v$ of the first change of state. This yields

$$
\begin{aligned}
& m_{l}(\theta, z, x)=\frac{d}{d x} \int_{0}^{x / c(l)} e^{-[\gamma(l)+\theta] v} \beta(l) z d v \\
& \quad+\int_{0}^{x / c(l)} e^{-[\gamma(l)+\theta] v} \mu(l)\left(\int_{0}^{x-c(l) v} m_{l+1}(\theta, z, y) m_{l}(\theta, z, x-c(l) v-y) d y\right) d v .
\end{aligned}
$$

with $m_{N}(\theta, z, x) \equiv 0$ after having implicitly taken the derivation in the second integral. Under the change of variable $w=x-c(l) v$, it becomes

$$
\begin{aligned}
m_{l}(\theta, z, x)= & \frac{\beta(l)}{c(l)} z e^{-\kappa(l) x} \\
& +\frac{\mu(l)}{c(l)} e^{-\kappa(l) x} \int_{0}^{x} e^{\kappa(l) w}\left(\int_{0}^{w} m_{l+1}(\theta, z, y) m_{l}(\theta, z, w-y) d y\right) d w,
\end{aligned}
$$

where $\kappa(l)=(\gamma(l)+\theta) / c(l)$ as before. Differentiating with respect to $x$, we get

$$
\frac{d}{d x} m_{l}(\theta, z, x)=-\kappa(l) m_{l}(\theta, z, x)+\frac{\mu(l)}{c(l)} \int_{0}^{x} m_{l+1}(\theta, z, y) m_{l}(\theta, z, x-y) d y .
$$

Multiplying both sides of this equality by $\exp (U x)$ and integrating over $x \in(0, \infty)$ then gives using (40)

$$
-m_{l}(\theta, z, 0) I-M_{l}(\theta, z) U=-\kappa(l) M_{l}(\theta, z)+\frac{\mu(l)}{c(l)} M_{l+1}(\theta, z) M_{l}(\theta, z) .
$$

Finally, since $m_{l}(\theta, z, 0)=[\beta(l) / c(l)] z$, we deduce that

$$
M_{l}(\theta, z)[\kappa(l) I-U]=\frac{\beta(l)}{c(l)} z I+\frac{\mu(l)}{c(l)} M_{l+1}(\theta, z) M_{l}(\theta, z),
$$

which is equivalent to (36) using the expression (34) of $U$.

Note that the inverse matrix in (36) is of the form $(c I-A)^{-1}$ where $c>0$ and $A$ is a (defective) generator matrix. Thus, the eigenvalues of $A$ are non-negative, so that the matrix $c I-A$ is invertible.

The vectors $\boldsymbol{\Psi}_{s}(\theta, z)$ can indeed be calculated from Proposition 3.2. Different methods allow to solve numerically the fixed-point equation (35). One possibility, for example, is to proceed iteratively as follows. First, $\boldsymbol{\Psi}_{s_{*}}(\theta, z)$ is evaluated as the limit

$$
\boldsymbol{\Psi}_{s_{*}}(\theta, z)=\lim _{k \rightarrow \infty} \Psi_{s_{*}}^{(k)}(\theta, z),
$$

where $\boldsymbol{\Psi}_{s_{*}}^{(0)}(\theta, z) \equiv \mathbf{0}$ and $\boldsymbol{\Psi}_{s_{*}}^{(k+1)}(\theta, z), k \geq 0$, is given by the equation

$$
\left(\boldsymbol{\Psi}_{s_{*}}^{(k+1)}(\theta, z)\right)_{j}=\int_{0}^{\infty} m_{s_{*}}(\theta, z, x) \Phi_{s_{*}-1, j}^{(k)}(\theta, z, x) d x,
$$


which is a simple modification of (38) and where, analogously to (30), (34),

$$
\Phi^{(k)}(\theta, z, x)=\exp \left(U^{(k)} x\right) \text {, with } U^{(k)}=Q(\theta, z)+\frac{\mu\left(s_{*}-1\right)}{c\left(s_{*}-1\right)} \mathbf{e}^{\prime} \Psi_{s_{*}}^{(k)}(\theta, z) .
$$

This leads to the formula

$$
\boldsymbol{\Psi}_{s_{*}}^{(k+1)}(\theta, z)=\mathbf{e} M_{s_{*}}^{(k)}(\theta, z),
$$

where the matrices $M_{l}^{(k)}(\theta, z), s_{*} \leq l \leq N-1$, are given as in (36) by

$$
M_{l}^{(k)}(\theta, z)=\frac{\beta(l)}{c(l)} z\left(\frac{\gamma(l)+\theta}{c(l)} I-Q(\theta, z)-\frac{\mu\left(s_{*}-1\right)}{c\left(s_{*}-1\right)} \mathbf{e}^{\prime} \Psi_{s_{*}}^{(k)}(\theta, z)-\frac{\mu(l)}{c(l)} M_{l+1}^{(k)}(\theta, z)\right)^{-1},
$$

with $M_{N}^{(k)}(\theta, z) \equiv 0$. The convergence of the iterative scheme then follows from probabilistic interpretations of the vectors $\boldsymbol{\Psi}_{s_{*}}^{(k)}(\theta, z)$ (see Bean et al. (2005)). Once $\boldsymbol{\Psi}_{s_{*}}(\theta, z)$ is obtained from (41), (42), the other vectors $\boldsymbol{\Psi}_{s}(\theta, z)$ can be easily calculated from (35), (36).

In the illustrations of Section 6 , we will essentially determine the probability that ruin occurs, i.e.

$$
P\left(T_{r}<T \mid S_{0}=n, R_{0}=u\right) .
$$

Obviously, this probability is obtained by setting $\theta=0$ and $z=1$ in (26), (27) and by summing over all possible states $j$ of $S_{T_{r}}$. In addition, the vectors involved $\boldsymbol{\Psi}_{s}(0,1)$ defined by (24) as

$$
\left(\Psi_{s}(0,1)\right)_{j}=P\left(S_{T_{r}}=j, T_{r}<T \mid S_{0}=s, R_{0}=0\right),
$$

are then calculated from Proposition 3.2 using the iterative procedure above.

\section{Contract with Lump-Sum Benefit}

In this section, we assume that the company pays each infected a lump-sum compensation which depends on the number of susceptible still present.

The Insurance Plan Specifically, when an infected person recovers from the disease, they receive one-time coverage of random, possibly constant, amount from the company. These lump-sums are independent variables and their distribution is function of the number of susceptibles at the time of recovery. As before, the company collects premiums continuously over time at the rate $p(s)$ when $S_{t}=s$. It is natural to take $p(0)=0$ and $p(s)>0$ when $s>0$, but these assumptions can be relaxed.

A different model might be for a person to receive a lump-sum once they contract the disease. In fact, the study of such a model is very similar to the one below. In this case, however, the initial infectious are not covered, which explains our choice.

To get the fair value of the premium, we again use the Equivalence Principle and require that

$$
\mathbb{E}\left[\int_{0}^{T} p\left(S_{t}\right) d t\right]=\mathbb{E}\left[\sum_{k=0}^{N-1} \sum_{l=1}^{\mathcal{M}_{T}^{(k)}} \mathcal{E}_{l, k}\right]
$$


where $\mathcal{M}_{T}^{(k)}$ is the total number of transitions from $k$ to $k+1$ susceptibles, and the $\mathcal{E}_{l, k}$ represent the lump-sums which are independent random variables with mean $\tilde{\alpha}(k)$ (that depends on $k$ as announced). This yields

$$
\mathbb{E}\left[\int_{0}^{T} p\left(S_{t}\right) d t\right]=\sum_{k=0}^{N-1} \tilde{\alpha}(k) \mathbb{E}\left[\mathcal{M}_{T}^{(k)}\right] .
$$

Now, it is clear that $\mathcal{M}_{T}^{(N-1)}=1$ a.s., and for $k<N-1$,

$$
\mathbb{E}\left[\mathcal{M}_{T}^{(k)} \mid S_{0}=s\right]=\mathbb{P}\left(\tau_{k}<T \mid S_{0}=s\right) \mathbb{E}\left[\mathcal{M}_{T}^{(k)} \mid S_{0}=k\right],
$$

where $\tau_{k}$ is the first-passage time at the height $k$ (see (7)). Moreover, we observe that $\left[\mathcal{M}_{T}^{(k)} \mid S_{0}=k\right]$ has a geometric distribution on $\{1,2, \ldots\}$ with success probability given by $\mathbb{P}\left(\tau_{k-1}>T \mid S_{0}=k\right)$. Note that this probability corresponds to $1-q_{k}$ where $q_{k}=q_{k}(0,1)$ is given by (17). Therefore,

$$
\mathbb{E}\left[\mathcal{M}_{T}^{(k)} \mid S_{0}=k\right]=\frac{1}{1-q_{k}}, \quad 0 \leq k \leq N-2 .
$$

Let $n$ be the initial number of susceptibles. From (45), (46) and applying Corollary 2.2 , we can then reexpress the equality (44) as

$$
\begin{aligned}
& \mathbb{E}\left[\int_{0}^{T} p\left(S_{t}\right) d t \mid S_{0}=n\right] \\
& =\tilde{\alpha}(N-1)+\sum_{k=0}^{n-1} \tilde{\alpha}(k) \mathbb{E}\left[\mathcal{M}_{T}^{(k)} \mid S_{0}=n\right]+\sum_{k=n}^{N-2} \tilde{\alpha}(k) \mathbb{E}\left[\mathcal{M}_{T}^{(k)} \mid S_{0}=n\right] \\
& =\tilde{\alpha}(N-1)+\sum_{k=0}^{n-1} \tilde{\alpha}(k) \frac{q_{k+1} \ldots q_{n}}{1-q_{k}}+\sum_{k=n}^{N-2} \tilde{\alpha}(k) \frac{1}{1-q_{k}} .
\end{aligned}
$$

In the special case where $\tilde{\alpha}(k)=\tilde{\alpha}$ for all $k$, i.e. the lump-sum distribution is the same for all infected, (43) reduces to

$$
\mathbb{E}\left[\int_{0}^{T} p\left(S_{t}\right) d t\right]=\mathbb{E}\left[\sum_{k=1}^{N-S_{0}+\mathcal{N}_{T}} \mathcal{E}_{k}\right],
$$

where $\mathcal{N}_{T}$ is the counting variable of Section 2 and the $\mathcal{E}_{k}$ are i.i.d. random variables with mean $\tilde{\alpha}$. For $S_{0}=n,(47)$ is replaced by

$$
\mathbb{E}\left[\int_{0}^{T} p\left(S_{t}\right) d t \mid S_{0}=n\right]=\tilde{\alpha}\left(N-n+\mathbb{E}\left[\mathcal{N}_{T} \mid S_{0}=n\right]\right),
$$

and from (9), (10), this becomes

$$
\sum_{l=n}^{N-1} \sum_{k=0}^{l} \frac{p(k)}{\mu(k)}\left(\prod_{j=k+1}^{l} \frac{\beta(j)}{\mu(j)}\right)=\tilde{\alpha}(N-n)+\tilde{\alpha} \sum_{l=n}^{N-1} \sum_{k=1}^{l}\left(\prod_{j=k}^{l} \frac{\beta(j)}{\mu(j)}\right) .
$$

In particular, if $p(s)=p s$, the fair premium level $p_{*}$ given by (48) is 


$$
p_{*}=\left[\tilde{\alpha}(N-n)+\tilde{\alpha} \sum_{l=n}^{N-1} \sum_{k=1}^{l}\left(\prod_{j=k}^{l} \frac{\beta(j)}{\mu(j)}\right)\right] /\left[\sum_{l=n}^{N-1} \sum_{k=1}^{l} \frac{k}{\mu(k)}\left(\prod_{j=k+1}^{l} \frac{\beta(j)}{\mu(j)}\right)\right] .
$$

The Reserve Process Let us introduce the process $\left\{R_{t}, t \geq 0\right\}$ where $R_{t}$ is the net capital of the insurance at time $t$, starting from $R(0)=u \geq 0$. Instead of (22), we have

$$
R_{t}=u+\int_{0}^{t} p\left(S_{v}\right) d v-\sum_{k=0}^{N-1} \sum_{l=1}^{\mathcal{M}_{t}^{(k)}} \mathcal{E}_{l, k}, \quad t \geq 0
$$

So, $R_{t}$ increases in a continuous, piecewise linear way with independent jumps downwards.

In the following, we assume that the lump sums $\mathcal{E}_{l, k}$ are distributed exponentially with the parameter $\alpha(k)(=1 / \tilde{\alpha}(k)$ above $)$. This case is less burdensome to deal with mathematically and allows us to use reasoning similar to that of Section 3. However, it does not cover lump-sums which would be constant, for example. To face this case, we can extend the model to more general jumps of phase type and then particularize to suitably normalized Erlang distributions. A different method consists in working with the Laplace transform of the probabilities of ruin with respect to the initial reserve $u$. The lump-sums can then be of arbitrary distribution but this approach leads to invert transforms of transforms numerically.

Define $T_{r}$ as the ruin time of the company when this actually occurs. Note that there may be a jump just before the end $T$ (compensation given to the only infected present), and this jump could cause the ruin at $T$, so that $T_{r} \leq T$. Let $\mathcal{N}_{T_{r}}$ be the total number of infections up to time $T_{r}$ and $S_{T_{r}}$ the number of susceptibles at time $T_{r}-$, i.e. just before $T_{r}$. We want to determine the expectation

$$
\mathbb{E}\left[e^{-\theta T_{r}} z^{\mathcal{N}_{T_{r}}} \mathbb{1}_{S_{T_{r}}=j, T_{r} \leq T}\right],
$$

for $\theta \geq 0, z \in(0,1]$ and $0 \leq j \leq N-1$.

For that, we define as in (24) a $N \times N$ matrix $\Psi(\theta, z)$ whose row vectors $\Psi_{s}(\theta, z)$ are of $j$-th element

$$
\left(\Psi_{s}(\theta, z)\right)_{j}=\mathbb{E}\left[e^{-\theta T_{r}} z^{\mathcal{N}_{T_{r}}} \mathbb{1}_{S_{T_{r}}=j, T_{r} \leq T} \mid S_{0}=s, R_{0}=0\right],
$$

for $0 \leq s, j \leq N-1$, and also the two following $N \times N$ matrices

$$
\Delta_{\alpha}=\left[\begin{array}{ccccc}
\alpha(0) & 0 & 0 & \cdots & 0 \\
0 & \alpha(1) & 0 & \cdots & 0 \\
0 & 0 & \alpha(2) & \cdots & 0 \\
\vdots & \vdots & \vdots & \ddots & \vdots \\
0 & 0 & 0 & \cdots & \alpha(N-1)
\end{array}\right], \quad E=\left[\begin{array}{ccccccc}
0 & 1 & 0 & 0 & \cdots & 0 & 0 \\
0 & 0 & 1 & 0 & \cdots & 0 & 0 \\
0 & 0 & 0 & 1 & \cdots & 0 & 0 \\
\vdots & \vdots & \vdots & \vdots & & \vdots & \vdots \\
0 & 0 & 0 & 0 & \cdots & 0 & 1 \\
0 & 0 & 0 & 0 & \cdots & 0 & 0
\end{array}\right] .
$$

Proposition 4.1 Let $\theta \geq 0, z \in(0,1]$ and $0 \leq j \leq N-1$. Starting with $n<N$ susceptibles and a reserve $u \geq 0$, 


$$
\mathbb{E}\left[e^{-\theta T_{r}} z^{\mathcal{N}_{T_{r}}} \mathbb{1}_{S_{T_{r}}=j, T_{r} \leq T}\right]=\left[\Psi(\theta, z) e^{\left(\Delta_{\alpha} E \Psi(\theta, z)-\Delta_{\alpha}\right) u}\right]_{n, j} .
$$

Proof As for (29), we get

$$
\mathbb{E}\left[e^{-\theta T_{r}} z^{\mathcal{N}_{T_{r}}} \mathbb{1}_{S_{T_{r}}=j, T_{r} \leq T}\right]=(\Psi(\theta, z) \Phi(\theta, z, u))_{n, j},
$$

where $\Psi(\theta, z)$ is the matrix defined trough $(50)$ and $(\Phi(\theta, z, x))_{s, j}$ is the transform

$$
\mathbb{E}\left[e^{-\theta T_{r}} z^{\mathcal{N}_{T_{r}}} \mathbb{1}_{S_{T_{r}}=j, T_{r} \leq T} \mid R_{0}=x\right],
$$

given that the susceptible process starts from $s$ with an immediate jump (exponentially distributed with parameter $\alpha(s)$ ) followed by a transition to $s+1$.

It is easy to see that as in (30),

$$
\Phi(\theta, z, x)=e^{U(\theta, z) x},
$$

for some $N \times N$ matrix $U(\theta, z)$. To determine $U(\theta, z)$, we first condition in (54) on the size of the initial jump, hence

$$
(\Phi(\theta, z, x))_{s, j}=\delta_{s, j} e^{-\alpha(s) x}+1_{s<N-1} \int_{0}^{x} \alpha(s) e^{-\alpha(s) y}\left(\boldsymbol{\Psi}_{s+1}(\theta, z) \Phi(\theta, z, x-y)\right)_{j} d y,
$$

where $1_{a}$ denotes the indicator function for a fixed set $a$. Using the notation (51), we can rewrite this in matrix form as

$$
e^{U(\theta, z) x}=e^{-\Delta_{\alpha} x}\left(I+\int_{0}^{x} \Delta_{\alpha} e^{\Delta_{\alpha} y} E \Psi(\theta, z) e^{U(\theta, z) y} d y\right) .
$$

Now, it suffices to differentiate both sides and take $x=0$ to deduce that

$$
U(\theta, z)=-\Delta_{\alpha}+\Delta_{\alpha} E \Psi(\theta, z) .
$$

The formula (52) then follows from (53), (55), (56).

Of course, it remains to determine the matrix $\Psi(\theta, z)$. Define the $N$ row vectors $\mathbf{e}_{0}, \ldots, \mathbf{e}_{N-1}$ where $\mathbf{e}_{l}=(0, \ldots, 1, \ldots, 0)$ with the 1 at the $(l+1)$-th position.

Proposition 4.2 $\Psi(\theta, z)$ is the minimal non-negative solution of the equation

$$
\boldsymbol{\Psi}_{s}(\theta, z)=\sum_{l=1}^{s} z^{s-l} \frac{\mu(l)}{\beta(l)} \mathbf{e}_{l}\left(\prod_{j=l}^{s} \boldsymbol{A}_{j}(\theta, z)\right)^{-1}+z^{s} \frac{\mu(0)}{\mu(0)+\theta} \mathbf{e}_{0}\left(\prod_{j=1}^{s} \boldsymbol{A}_{j}(\theta, z)\right)^{-1},
$$

for $0 \leq s \leq N-1$, where

$$
\boldsymbol{A}_{j}(\theta, z)=\frac{1}{\beta(j)}\left[(\gamma(j)+\theta) I+p(j) \Delta_{\alpha}-p(j) \Delta_{\alpha} E \Psi(\theta, z)\right] .
$$

Proof For an epidemic starting with $s=0$, we have $(\Psi(\theta, z))_{0, j}=\delta_{j, 0} \mathbb{E}\left[\exp \left(-\theta \tau_{0}\right)\right]$, so that 


$$
\mathbf{\Psi}_{0}(\theta, z)=\frac{\mu(0)}{\mu(0)+\theta} \mathbf{e}_{0}
$$

which corresponds to (57) when $s=0\left(\sum_{l=1}^{0} \equiv 0, \prod_{j=1}^{0} \equiv 1\right)$. Consider now the case with $s>0$. To find $(\Psi(\theta, z))_{s, j}$, we examine all the trajectories of the susceptible process which are possible between $t=0$ and the first time when the number of susceptibles increases. These trajectories are in number $l+1$ and of the form $s \rightarrow s-1 \rightarrow \cdots \rightarrow l \rightarrow l+1$ for $l=0, \ldots, s$. Indeed, for such a $l$, the number of susceptibles first decreases from $s$ to $l$ (without recovery in the interval), then a first recovery occurs which means a transition from $l$ to $l+1$. With the previous notation $\kappa(s)$ adapted here to $\kappa(s)=(\gamma(s)+\theta) / p(s), s>0$, we then obtain

$$
\begin{gathered}
(\Psi(\theta, z))_{s, j} \\
=\sum_{l=1}^{s} \int_{0}^{\infty} e^{-\kappa(s) y_{s}} \frac{\beta(s)}{p(s)} z d y_{s} \int_{0}^{\infty} e^{-\kappa(s-1) y_{s-1}} \frac{\beta(s-1)}{p(s-1)} z d y_{s-1} \cdots \\
\ldots \int_{0}^{\infty} e^{-\kappa(l) y_{l}} \frac{\mu(l)}{p(l)} d y_{l}\left(\Phi\left(\theta, z, y_{s}+y_{s-1}+\cdots+y_{l}\right)\right)_{l, j} \\
+\int_{0}^{\infty} e^{-\kappa(s) y_{s}} \frac{\beta(s)}{p(s)} z d y_{s} \int_{0}^{\infty} e^{-\kappa(s-1) y_{s-1}} \frac{\beta(s-1)}{p(s-1)} z d y_{s-1} \cdots \\
\cdots \int_{0}^{\infty} e^{-[\mu(0)+\theta] y_{0}} \mu(0) d y_{0}\left(\Phi\left(\theta, z, y_{s}+y_{s-1}+\cdots+y_{1}\right)\right)_{0, j} .
\end{gathered}
$$

Using the exponential form (55) for $\Phi(\theta, z, x)$, this equality gives

$$
\begin{aligned}
\Psi_{s}(\theta, z)= & \sum_{l=1}^{s} \mathbf{e}_{l}\left(z^{s-l} \prod_{j=l+1}^{s} \frac{\beta(j)}{p(j)} \int_{0}^{\infty} e^{-[\kappa(j) I-U] y} d y\right) \frac{\mu(l)}{p(l)} \int_{0}^{\infty} e^{-[\kappa(l) I-U] y} d y \\
& +\mathbf{e}_{0}\left(z^{s} \prod_{j=1}^{s} \frac{\beta(j)}{p(j)} \int_{0}^{\infty} e^{-[\kappa(j) I-U] y} d y\right) \mu(0) \int_{0}^{\infty} e^{-[\mu(0)+\theta] y} d y \\
= & \sum_{l=1}^{s} z^{s-l} \frac{\mu(l)}{\beta(l)} \mathbf{e}_{l}\left(\prod_{j=l}^{s} \frac{\beta(j)}{p(j)}[\kappa(j) I-U]^{-1}\right) \\
& +z^{s} \frac{\mu(0)}{\mu(0)+\theta} \mathbf{e}_{0}\left(\prod_{j=1}^{s} \frac{\beta(j)}{p(j)}[\kappa(j) I-U]^{-1}\right)
\end{aligned}
$$

in which the inverse matrices exist as for (36). Substituting (56) for $U \equiv U(\theta, z)$, (59) leads to the formulas (57), (58). That $\Psi(\theta, z)$ corresponds to the minimal non-negative solution of (57) can be deduced using standard arguments (see e.g. Bean et al. (2005)).

Analogously to Section 3, different techniques allow the numerical evaluation of $\Psi(\theta, z)$. For example, this matrix can be obtained as the limit

$$
\Psi(\theta, z)=\lim _{k \rightarrow \infty} \Psi^{(k)}(\theta, z),
$$

where $\Psi^{(0)}(\theta, z)=0$ and $\Psi_{s}^{(k+1)}(\theta, z), k \geq 0$, is given by the following recursion from (57), (58) 


$$
\boldsymbol{\Psi}_{s}^{(k+1)}(\theta, z)=\sum_{l=1}^{s} z^{s-l} \frac{\mu(l)}{\beta(l)} \mathbf{e}_{l}\left(\prod_{j=l}^{s} \boldsymbol{A}_{j}^{(k)}(\theta, z)\right)^{-1}+z^{s} \frac{\mu(0)}{\mu(0)+\theta} \mathbf{e}_{0}\left(\prod_{j=1}^{s} \boldsymbol{A}_{j}^{(k)}(\theta, z)\right)^{-1}
$$

for $0 \leq s \leq N-1$, where

$$
\boldsymbol{A}_{j}^{(k)}(\theta, z)=\frac{1}{\beta(j)}\left[(\gamma(j)+\theta) I+p(j) \Delta_{\alpha}-p(j) \Delta_{\alpha} E \Psi^{(k)}(\theta, z)\right] .
$$

In particular, the probability that the ruin will occur, i.e. $P\left(T_{r}<T \mid S_{0}=n, R_{0}=u\right)$, follows from (52) with $\theta=0$ and $z=1$, after having summed over all possible states $j$ of $S_{T_{r}}$. The unknown matrix $\Psi(0,1)$ is then evaluated from Proposition 4.2 via the previous recursion.

With Annuity Benefit An insurance contract can provide for both an annuity and lumpsum benefits. Under the same notation as before, the fair premium level is obtained from (19) and (43) by

$$
\mathbb{E}\left[\int_{0}^{T} p\left(S_{t}\right) d t\right]=\mathbb{E}\left[\int_{0}^{T} r\left(S_{t}\right) d t\right]+\mathbb{E}\left[\sum_{k=0}^{N-1} \sum_{l=1}^{\mathcal{M}_{T}^{(k)}} \mathcal{E}_{l, k}\right] .
$$

Moreover, the last expectation in (60) is still given by (44) with the right-hand side of (47).

Combining (22) and (49), the associated reserve process is defined as

$$
R_{t}=u+\int_{0}^{t} p\left(S_{v}\right) d v-\int_{0}^{t} r\left(S_{v}\right) d v-\sum_{k=0}^{N-1} \sum_{l=1}^{\mathcal{M}_{t}^{(k)}} \mathcal{E}_{l, k}, \quad t \geq 0
$$

When the variables $\mathcal{E}_{l, k}$ are distributed exponentially in (61), the problem of ruin can be analyzed by distinguishing the cases where the ruin is due to the payment of a lump-sum or not, then adapting the previous reasoning. This leads to heavy writing in the formulas, and we do not report the results here for the sake of brevity.

\section{Regenerative Epidemic Episodes}

A company could offer an insurance contract for normal times as well as additional coverage for epidemic episodes. In the current framework, this would lead to examining an insurance contract for a regenerative extension of the SIS type model. The objective of this section is precisely to study such a process, this time using a Brownian reserve process.

More precisely, we consider a risk process involving a closed population of $N$ individuals and which alternates between two regimes, normal or epidemic.

- In the normal regime, there is no spread of infection, so everyone is in good health (with regard to the infectious disease). The reserve then evolves as a Brownian motion of drift $c(N)$ and of variance $\sigma^{2}(N)>0$.

- In the epidemic regime, part of the population becomes infected and transmits the disease to others through an SIS schema. The evolution of reserve is here also described by a Brownian motion but its parameters now depend on the state of the 
population: when there are $s$ susceptibles present, the drift of the Brownian motion is $c(s)$ and its variance is $\sigma^{2}(s)>0$.

During a normal regime, an epidemic can occur at the rate $\lambda_{e}$. If this happens, a certain number of people are instantly infected: these are the $I_{0}$ infectives of the epidemic. So, the number of starting susceptibles $S_{0}=N-I_{0}$ is a random variable of distribution $\pi$ where

$$
\pi_{l}=\mathbb{P}\left(S_{0}=l\right), \quad 0 \leq l \leq N-1 .
$$

The model thus switches to the epidemic regime, and it will remain in this situation until the end of the epidemic, i.e. when the $N$ individuals are all susceptible. Then, it returns to the normal regime until the next outbreak, and so on.

Consequently, the susceptible process $\{S(t), t \geq 0\}$ is described by a Markov-modulated Brownian motion whose generator is the matrix $T$ of dimension $(N+1) \times(N+1)$ given by

$$
T=\left[\begin{array}{ccccccc}
-\gamma(0) & \mu(0) & 0 & 0 & \cdots & 0 & 0 \\
\beta(1) & -\gamma(1) & \mu(1) & 0 & \cdots & 0 & 0 \\
0 & \beta(2) & -\gamma(2) & \mu(2) & \cdots & 0 & 0 \\
\vdots & \vdots & \vdots & \vdots & \ddots & \vdots & \vdots \\
0 & 0 & 0 & 0 & \cdots & -\gamma(N-1) & \mu(N-1) \\
\lambda_{e} \pi_{0} & \lambda_{e} \pi_{1} & \lambda_{e} \pi_{2} & \lambda_{e} \pi_{3} & \cdots & \lambda_{e} \pi_{N-1} & -\lambda_{e}
\end{array}\right],
$$

where, as before, $\beta(s)$ and $\mu(s)$ are the global infection and recovery rates in presence of $s$ susceptibles. For $s<N$, we set $\gamma(s)=\mu(s)+\beta(s)$ with $s<N$, all the other rates being strictly positive. Note that the state $N$ is no longer absorbing. Also, when $\lambda_{e}=0$, this model is a variant of the one in Section 3 in which the reserve process is now Brownian instead of piecewise linear.

The Insurance Plan The reserve evolves as a Brownian motion of drift and variance depending on the state of the population. When $S_{t}=s$, the drift $c(s)$ roughly represents the premium rate $p(s)$ minus the reimbursement rate $r(s)$. It is natural to assume that $c(s)$ increases with $s$, but no further assumption is required here. The variance $\sigma^{2}(s)$ takes into account the relative uncertainty in premium collection and care payments. For example, it could be set independently of $s$, or be of the form $\sigma_{1}^{2} s+\sigma_{2}^{2}(N-s)$ if each susceptible or infective generates some noise.

A central problem for insurance is determining a premium rate $p(s)$ given a premium structure. In a simplified approach, it is reasonable to assume that the hedge rates $r(s)$ and the variances $\sigma^{2}(s)$ can be estimated by the company. A priori, there are (at least) two different ways to set the premium.

A first criterion consists in applying the Equivalence Principle to each epidemic period. So, every time an epidemic is triggered, the expected net gain for the company must be equal to 0 . This implies that the fair premium satisfies the condition

$$
\mathbb{E}\left[\int_{0}^{T} p\left(S_{t}\right) d t \mid S_{0} \sim \pi\right]=\mathbb{E}\left[\int_{0}^{T} r\left(S_{t}\right) d t \mid S_{0} \sim \pi\right] .
$$

Another criterion is to ask that the mean stationary drift of the reserve process, $d_{\star}$, is equal to 0 . An advantage here is that episodes under normal regime are taken into account 
and the expected net gain of the company is 0 in the long run. In this case, the fair premium satisfies the equation

$$
d_{\star} \equiv \sum_{s=0}^{N} c(s) v_{s}=0
$$

where the vector $v$ is the stationary distribution for the generator $T$ defined in (62) (i.e. such that $\boldsymbol{v} T=\mathbf{0})$.

The Reserve Process Consider the problem of ruin with this Brownian regenerative epidemic process. Ruin is generally not certain, in fact it will occur almost surely in finite time iff $d_{\star} \leq 0$. We will determine a transform similar to the one in the previous sections but including an additional random variable $\mathcal{J}_{T_{r}}$ which counts the epidemics triggered before the ruin. For convenience of notation, set $\gamma(N)=\lambda_{e}$ and define, for $\mathcal{J}_{T_{r}}$,

$$
\omega_{s}=\frac{c(s)}{\sigma^{2}(s)}+\sqrt{\frac{c(s)^{2}}{\sigma^{4}(s)}+\frac{2(\gamma(s)+\theta)}{\sigma^{2}(s)}}, \quad \eta_{s}=\frac{-c(s)}{\sigma^{2}(s)}+\sqrt{\frac{c(s)^{2}}{\sigma^{4}(s)}+\frac{2(\gamma(s)+\theta)}{\sigma^{2}(s)}} .
$$

Proposition 5.1 Let $\theta \geq 0$ and $z, w \in(0,1]$. Starting with $n$ susceptibles and a reserve $u \geq 0$,

$$
\mathbb{E}\left[e^{-\theta T_{r}} z^{\mathcal{N}_{T_{r}}} w^{\mathcal{J}_{T_{r}}} \mathbb{1}_{S_{T_{r}}=j, T_{r}<\infty}\right]=\left(e^{U(\theta, z, w) u}\right)_{n, j},
$$

where $U(\theta, z, w) \equiv U$ is a $N+1 \times N+1$ matrix of elements $U_{s, j}, 0 \leq s, j \leq N$, which can be obtained as the limit of the matrix sequence $U^{(k)}, k \geq 0$, defined recursively as follows. First, $U^{(0)}=-\operatorname{diag}\left(\omega_{0}, \ldots, \omega_{N}\right)$, then for $k \geq 0$, when $0 \leq s<N$,

$$
\begin{gathered}
U_{s, j}^{(k+1)}=-\delta_{s, j} \omega_{s}+1_{s>0} \omega_{s} \eta_{s} \frac{\beta(s)}{\gamma(s)} z\left(\left[\eta_{s} I-U^{(k)}\right]^{-1}\right)_{s-1, j} \\
+w^{\delta_{s, 0}} \omega_{s} \eta_{s} \frac{\mu(s)}{\gamma(s)}\left(\left[\eta_{s} I-U^{(k)}\right]^{-1}\right)_{s+1, j},
\end{gathered}
$$

and when $s=N$,

$$
U_{N, j}^{(k+1)}=-\delta_{N, j} \omega_{N}+\omega_{N} \eta_{N} \sum_{l=0}^{N-1} \pi_{l}\left(\left[\eta_{N} I-U^{(k)}\right]^{-1}\right)_{l, j} .
$$

Proof We use the following standard result: if $\{B(t), t \geq 0\}$ is a Brownian motion with drift $c(s)$ and variance $\sigma^{2}(s)$, and $\tau$ is an independent exponential variable of parameter $\gamma(s)+\theta$, then the two random variables

$$
Z_{1}=-\inf _{0 \leq t \leq \tau} B(t), \quad \text { and } \quad Z_{2}=B(\tau)-\inf _{0 \leq t \leq \tau} B(t),
$$

are independent and exponential of respective parameters $\omega_{s}$ and $\eta_{s}$ given by (63) (see e.g. Corollary 45.8 in Sato (1999)).

The exponential form in the right hand side of (64) is justified as before. Now, in the iterative scheme above, the matrix $\exp \left(U^{(k)} x\right)$ gives the transform considered in (64) but when there are restrictions on the sample paths allowed until the end of the epidemic. These constraints are defined recursively for $k=0,1, \ldots$ as follows. 
First, the matrix $U^{(0)}$ is defined as

$$
\left(e^{U^{(0)} x}\right)_{s, j}=\mathbb{E}\left[e^{-\theta T_{r}} z^{\mathcal{N}_{T_{r}}} w^{\mathcal{J}_{T_{r}}} \mathbb{1}_{S_{T_{r}}=j, T_{r}<\tau}\right],
$$

where $\tau$ denotes the first transition time of the susceptible process. In other words, the additional constraint is that ruin must occur before any infection or recovery. We can easily see that $U^{(0)}=-\operatorname{diag}\left(\omega_{0}, \ldots, \omega_{N}\right)$ as indicated in the above statement.

Next, assuming $U^{(k)}$ known, we define $U^{(k+1)}, k \geq 0$, such that $\left(\exp \left(U^{(k+1)} x\right)\right)_{s, j}$ gives the transform (64) under new constraints. Starting from level $x$, the reserve process evolves normally until the first transition time of the susceptible process. During this period, the reserve process will have attained some minimum $x-Z_{1}$ before leaving at the higher level $x-Z_{1}+Z_{2}$ when the first infection or recovery occurs. Here, $Z_{1}$ and $Z_{2}$ are the random variables defined in (67) and which are independent and exponential of parameters $\omega_{s}$ and $\eta_{s}$, respectively. Then, the first return to level $x-Z_{1}$ must be done by one of the sample paths allowed by the restrictions imposed on $U^{(k)}$.

From this construction, we condition on $\left(Z_{1}, Z_{2}\right)=\left(y_{1}, y_{2}\right)$ to write that, when there are $0 \leq s<N$ initial susceptibles,

$$
\begin{aligned}
& \left(e^{U^{(k+1)} x}\right)_{s, j}=\delta_{s, j} e^{-\omega_{s} x} \\
& +1_{s>0} \int_{0}^{x} \int_{0}^{\infty} \omega_{s} e^{-\omega_{s} y_{1}} \eta_{s} e^{-\eta_{s} y_{2}} z \frac{\beta(s)}{\gamma(s)}\left(e^{U^{(k)} y_{2}} e^{U^{(k+1)}\left(x-y_{1}\right)}\right)_{s-1, j} d y_{1} d y_{2} \\
& +w^{\delta_{s, 0}} \int_{0}^{x} \int_{0}^{\infty} \omega_{s} e^{-\omega_{s} y_{1}} \eta_{s} e^{-\eta_{s} y_{2}} \frac{\mu(s)}{\gamma(s)}\left(e^{U^{(k)} y_{2}} e^{U^{(k+1)}\left(x-y_{1}\right)}\right)_{s+1, j} d y_{1} d y_{2},
\end{aligned}
$$

and when $s=N$,

$$
\begin{aligned}
\left(e^{U^{(k+1)} x}\right)_{N, j} & =\delta_{N, j} e^{-\omega_{N} x} \\
& +\int_{0}^{x} \int_{0}^{\infty} \omega_{N} e^{-\omega_{N} y_{1}} \eta_{N} e^{-\eta_{N} y_{2}} \sum_{l=0}^{N-1} \pi_{l}\left(e^{U^{(k)} y_{2}} e^{U^{(k+1)}\left(x-y_{1}\right)}\right)_{l, j} d y_{1} d y_{2} .
\end{aligned}
$$

It suffices then to differentiate each side of these two equations with respect to $x$ and to take $x=0$, which provides the announced formulas (65) and (66), the inverse matrices existing as for (36). Finally, the convergence of the algorithm follows directly from the physical interpretation of the constraints since the restrictions on the trajectories of the process disappear when $k$ goes to $\infty$.

\section{Some Numerical Examples}

By way of illustration, we examine in this section two particular epidemic models of SIS type. On the one hand, we will consider the classical logistic model where the overall infection and recovery rates are $\beta(s)=\beta s(N-s)$ and $\mu(s)=v(N-s)$. On the other hand, we will generalize this model by supposing the transmission rate per infective no longer as a linear function $\beta s$ of the number of susceptibles, but as a power function $\beta s^{b}$ with $b>0$ of this number. Such a rate makes it possible to quantify the capacity of the disease to contaminate a whole set of susceptibles. So, when $b<1$ (resp. $>1$ ), having more than fewer susceptibles gives more (resp. less) safety. Another extension in the same vein could be a power function $\beta(N-s)^{a}$ with $a>0$ for the transmission rate per susceptible. These two 

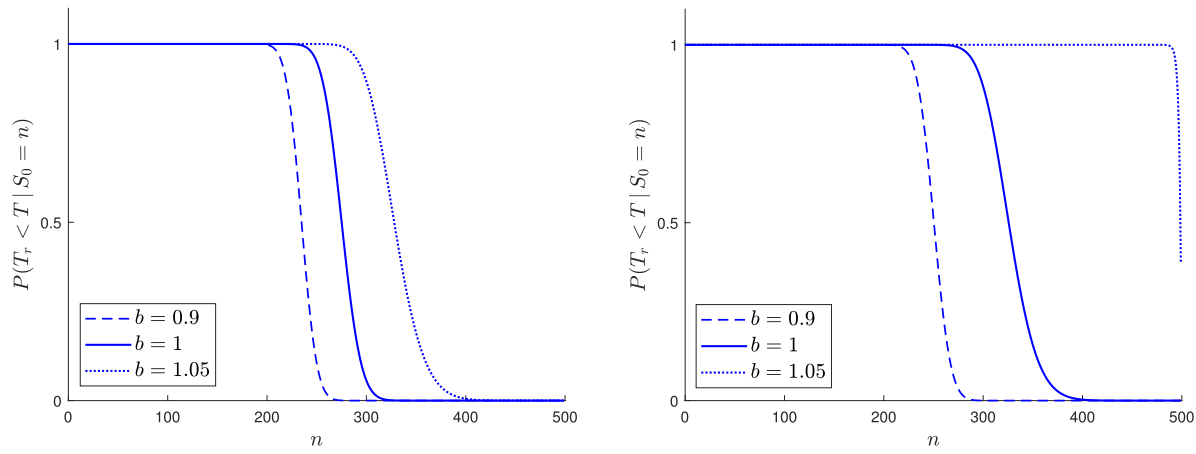

Fig. 1 Ruin probability as a function of the initial number $n$ of susceptibles in the annuity case for $b=0.9,1,1.05$ when $p=0.3, \rho=1, u=100$ and $v=1, N=500, \beta=0.9 / N$ (left) or $\beta=1.2 / N$ (right)

cases have already been proposed by Severo (1969) for a SIR model and have since been discussed in numerous papers (see e.g. Gouriéroux and $\mathrm{Lu}$ (2020) and their references).

The premium rates are assumed equal to $p$ per susceptible, thus $p(s)=p s$. We start with a contract providing for an annuity benefit (Section 3 ). The coverage rates are taken equal to $\rho$ per infective, thus $r(s)=\rho(N-s)$. Figure 1 gives the probability of ruin as a function of the number $n$ of initial susceptibles for $b=1$ (logistic), $b=0.9$ (more safety) and $b=1.05$ (less safety). The premium and coverage rates are $p=0.3$ and $\rho=1$ with an initial reserve $u=100$, and the recovery and infection coefficients are $v=1$ and $\beta=0.9 / N$ (left) or $\beta=1.2 / N$ (right) for a population size $N=500$. As expected, we see that a higher value of $b$ or $\beta$ results in a higher probability of ruin. Both graphs show that only a small range of $n$ (neither too small nor too large) leads to a non-extreme probability of ruin (other than 0 or 1$)$.

For a similar model with $b=1, b=0.9$ and $b=1.05$, Figure 2 gives the fair premium rate (left) and the corresponding probability of ruin (right) again as a function of $S_{0}=n$. The parameters here are $p_{\star}^{(n)}$ given by $(21), \rho=1, u=100$ and $v=1, \beta=1 / N, N=500$. We note that the fair premium depends rather strongly on the safety-in-number parameter $b$. However, for most values of $n$, the use of the fair premium rate leads to a very high probability of ruin. This is not really surprising since choosing the fair premium ensures that


Fig. 2 Fair premium rate (left) and corresponding ruin probability (right) as a function of the initial number $n$ of susceptibles in the annuity case for $b=0.9,1,1.05$ when $p=p_{\star}^{(n)}, \rho=1, u=100$ and $v=1, \beta=1 / N$, $N=500$ 
the net profit of the company over the entire epidemic will be zero. As the reserve increases sharply towards the end of the epidemic (when the number of susceptibles is close to $N$ ), it is normal that this is compensated earlier in the course of the epidemic by significant losses which will often result in the ruin.

Thus, the Equivalence Principle provides a level of premium which may be insufficient if the main objective is to avoid ruin (for relatively small initial reserve). Another way to set the premium is to ask that the probability of ruin does not exceed a fixed threshold. This is what is illustrated with Figure 3 in the case of the logistic model. The probability of ruin is drawn as a function of the premium coefficient $p$ when $u=1$ (left) or $u=100$ (right). The other parameters are $\rho=1$ and $\nu=1, \beta=1.2 / N, n=490, N=500$. We observe that the probability of ruin remains high and relatively constant until the premium $p$ approximately reaches the level of 0.2 , then this probability suddenly jumps to zero. In such a situation, a premium amount slightly greater than 0.2 would therefore be advisable and would make ruin very unlikely.

We finally compare the insurance risks for a contract in the logistic model with annuity benefit (Section 3) and with a lump sum benefit (Section 4) when an infected person receives the same coverage on average. Figure 4 gives the probability of ruin as a function of $n$ (left) when $u=100$, or as a function of $u$ when $n=350$ (right). The other parameters are $p=0.3, \rho=1, \alpha=1$ and $\nu=1, \beta=1.2 / N, N=500$. Note that $1 / \alpha=\rho / \nu$, i.e. the average reimbursement per infective is indeed the same in both models. A significant difference is that with the annuity, the cost of an infected person is directly related to his period of infection, while with the lump-sum, this cost is independent of the duration of the infection. This tends to result in a higher probability of ruin in the latter case, as illustrated by both graphs. Note that the effect of $n$ is again roughly all-or-nothing.

These examples use special parameter values that have been chosen to highlight different possible situations. In reality, these parameters must of course be estimated, which poses new and interesting research questions. In addition, the size of the population $N$ can be very large when considering a large company. The implementation of the formulas derived in the paper then takes a lot of time and approximation methods become useful, possibly necessary.

Further Discussions Here we continued our previous work on the problem of ruin for an infectious disease of the SIR type (Lefèvre and Simon 2021) by considering the important alternative of an infectious disease of the SIS type. The epidemic situation in these two models
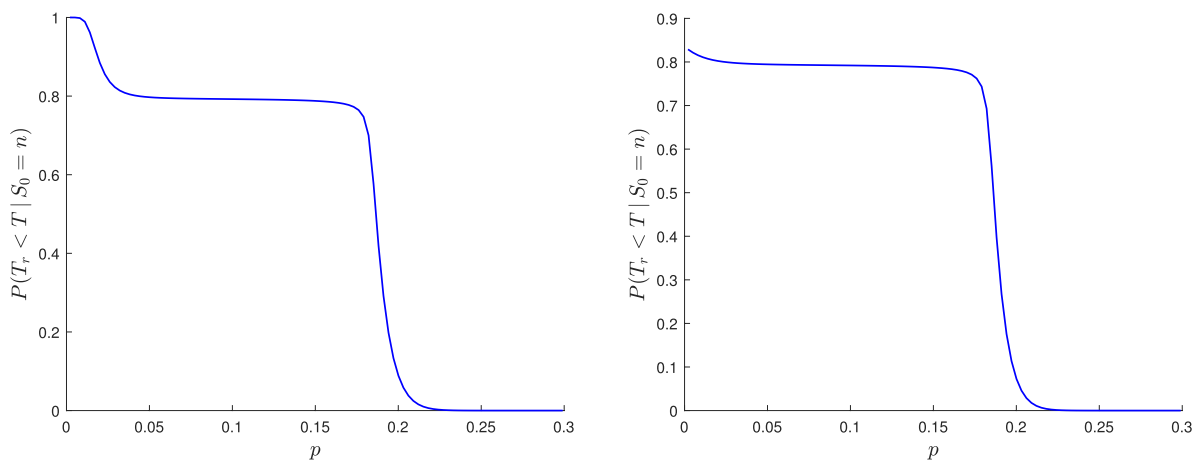

Fig. 3 Ruin probability as a function of the premium rate $p$ in the annuity case for $b=1$ when $\rho=1, u=1$ (left) or $u=100$ (right), and $v=1, \beta=1.2 / N, n=490, N=500$ 

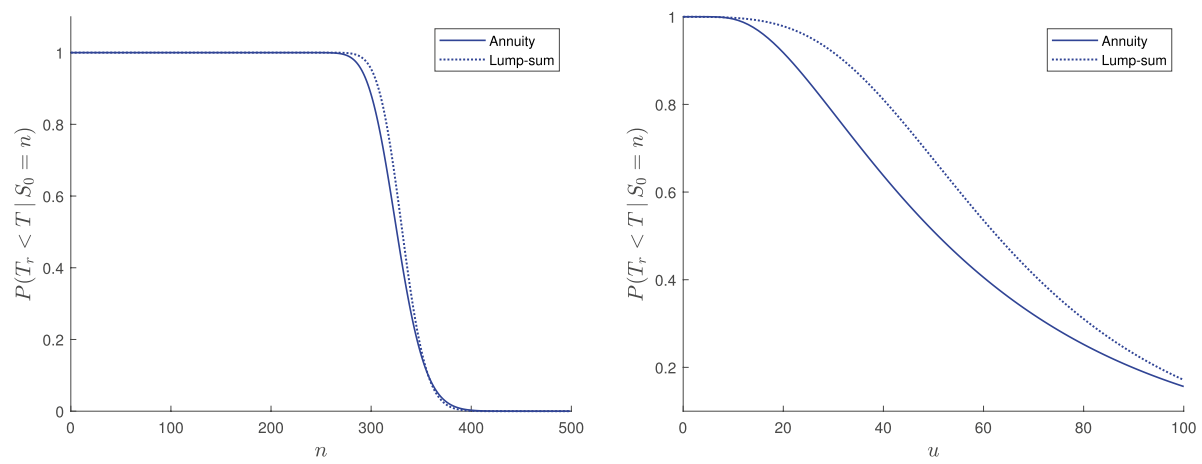

Fig. 4 Comparison of the ruin probability in the annuity and lump-sum cases for $b=1$ as a function of $n$ with $u=100$ (left) or a function of $u$ with $n=350$ (right), when $p=0.3, \rho=1, \alpha=1$ and $v=1$, $\beta=1.2 / N, N=500$

is very different since in the case of SIR, the epidemic ends with a certain number of remaining susceptibles, while in the case of SIS, the entire population is ultimately infected. Nevertheless, to study the ruin problem, we were able to use in both cases matrix-analytic methods and similar probabilistic tools. The analysis for the current model turns out to be a little less cumbersome, which allowed us to also deal with regenerative epidemic episodes.

Extensions of the modeling could take into account the intervention measures taken by the health authorities such as the isolation of infectives and the vaccination of susceptibles. It would certainly come in handy for practical purposes, especially now with the COVID-19 outbreak.

In actuarial science, it is normal to incorporate a discount factor into costs and premiums. As an epidemic generally does not last long, the question seems to be less relevant in this setting. However, such a factor could be used but would require working with non-homogeneous transition rates.

Acknowledgements We thank the referees and the associate editor for their useful comments and suggestions. C. Lefèvre received partial support from the Chair DIALog sponsored by CNP Assurances.

\section{References}

Andersson H, Britton T (2000) Stochastic Epidemic Models and their Statistical Analysis. Lecture Notes in Statistics 151, Springer, New York

Andersson H, Djehiche B (1998) A threshold limit theorem for the stochastic logistic epidemic. J Appl Probab 35:662-670

Asmussen S, Albrecher H (2010) Ruin Probabilities, 2nd edn. World Scientific, Singapore

Bean N, O'Reilly M, Taylor PG (2005) Hitting probabilities and hitting times for stochastic fluid flows. Stochastic Processes and their Applications 115(9):1530-1556

Britton T, Pardoux E (2019) Stochastic Epidemic Models with Inference. Lecture Notes in Mathematics 2255, Springer, Cham

Clancy D (2005) A stochastic SIS infection model incorporating indirect transmission. J Appl Probab 42:726-737

Clancy D, Tjia E (2018) Approximating time to extinction for endemic infection models. Methodol Comput Appl Probab 20:1043-1067

Daley D, Gani J (1999) Epidemic Modelling: an Introduction. Cambridge University Press, Cambridge

Dickson DCM (2017) Insurance Risk and Ruin, 2nd edn. Cambridge University Press, Cambridge

Feng R, Garrido J (2011) Actuarial applications of epidemiological models. North Am Act J 15:112-136 
Feng R, Garrido J, Jin L, Loke SH, Zhang L (2022) Epidemic compartmental models and their Insurance applications. In: Boado-Penas MC, Eisenberg J, Şahin Ş (eds) Pandemics : Insurance and Social Protection. Springer Actuarial, Springer, Cham, pp 13-40

Gouriéroux C, Lu Y (2020) SIR model with stochastic transmission. https://arxiv.org/abs/2011.07816

Lefèvre C, Picard P, Simon M (2017) Epidemic risk and insurance coverage. J Appl Probab 54:286-303

Lefèvre C, Simon M (2021) Ruin problems for epidemic insurance. Adv Appl Probab 53:484-509

Nåsell I (1999) On the time to extinction in recurrent epidemics. J Roy Stat Soc B 61:309-330

Nåsell I (2011) Extinction and Quasi-Stationarity in the Stochastic Logistic SIS Model. Lecture Notes in Mathematics 2022, Springer, Heidelberg

Sato K (1999) Lévy Processes and Infinitely Divisible Distributions. Cambridge University Press, Cambridge

Schmidli H (2018) Risk Theory. Springer, Cham

Severo NC (1969) Generalizations of some stochastic epidemic models. Math Biosci 4:395-402

van Doorn EA, Pollett PK (2013) Quasi-stationary distributions for discrete-state models. Eur J Oper Res 230:1-14

Publisher's Note Springer Nature remains neutral with regard to jurisdictional claims in published maps and institutional affiliations. 